\title{
Photoacoustic Study of Optical and Thermal Properties of CdTe Quantum Dots
}

\author{
A. Badawi ${ }^{1,2}$, N. Al-Hosiny ${ }^{1}$, S. Abdallah ${ }^{1,3}$, S. Negm $^{3}$ and \\ H. Talaat ${ }^{2}$ \\ ${ }^{1}$ Department of Physics, Faculty of Science, Taif University, Taif, Saudi \\ Arabia \\ ${ }^{2}$ Physics Department, Faculty of Science, Ain Shams University, \\ Abbassia Cairo, Egypt \\ ${ }^{3}$ Department of Mathematical and Physical Engineering, Faculty of \\ Engineering (Shoubra), Benha \\ University, Cairo, Egypt
}

The optical and thermal properties of CdTe quantum dots (QDs) of different particle size are measured using photoacoustic technique (PA). CdTe (QDs) were synthesized using the chemical deposition method (CD).The PA spectra show a blue shift as the sizes of CdTe QDs decrease which is in close agreement with that obtained by UV-Visible spectrophotometry. The sizes of the nano-particles were estimated using effective mass approximation (EMA) model giving radii ranging from $2.13 \mathrm{~nm}$ to $2.43 \mathrm{~nm}$. The estimated values are comparable to those obtained by transmission electron microscope (TEM). The thermal diffusivity ( $D)$, thermal effusivity (e), and thermal conductivity ( $k$ ) of all CdTe samples were also studied by PA technique. The determined values of $D$ and $k$ are at least one order of magnitude larger than the bulk value.

\section{Introduction:}

During the last two decades, research on quantum size semiconductor particles has increased enormously. This is due to their exciting novel properties such as optical, thermal and electronic properties, when they have size comparable to, or smaller than, the dimensions of the exciton within their corresponding bulk material, in particular, II -VI semiconductor nanoparticles are currently of great interests for their practical applications in a variety of optoelectronic devices such as, high efficiency thin film transistors, lightemitting diodes, electron-beam pumped lasers, electroluminescent devices and others [1-3]. Recently, CdTe Semiconductor nano crystals (i.e. quantum dots (QDs)) have become one of the most promising materials in many applications 
including high efficiency solar cell fabrication. Therefore, massive attention has been devoted to investigate its' thermal and optical properties in order to improve the performance of the solar cell devices and also pave the way for new applications [4]. In general, the absorption spectra can be measured by the conventional transmitted intensity method. However, in this method, samples should be sufficiently thin and have good quality surfaces through pretreatment. This requires samples to be placed in a matrix of transparent material which, on the other hand, would interfere with the measurements of thermal properties. In contrast, Photoacoustic technique offers a powerful tool for studing the optical, electronic and thermal properties of such materials in a noncontact and nondestructive manner. It has also been observed that the excitonic transitions of semiconductor nano crystals are well resolved in PA spectra as compared to the conventional optical absorption spectra [2]. In the present work, we report the size dependence of PA spectra for of CdTe QDs to investigate the effect of size confinement on the electronic states. Furthermore, PA technique is easily applied to determine the thermal properties of the CdTe QDs samples of different particles sizes.

\section{Experimental}

Colloidal CdTe nano crystals were synthesized by the method of Talapin et al [5].The synthesis was carried out as follows: Five grams of dodecylamine (DDA) were dissolved in $7 \mathrm{~mL}$ of tri-n-octylphosphine (TOP) at $50^{\circ} \mathrm{C}$ in a 50 $\mathrm{mL}$ two-neck flask with a reflux condenser attached. Subsequently, $0.11 \mathrm{~mL}$ $(1.47 \mathrm{mmol})$ of dimethylcadmium and $0.128 \mathrm{~g}(1 \mathrm{mmol})$ of tellurium powder were added under stirring, and the temperature was slowly increased to $180^{\circ} \mathrm{C}$. After $30 \mathrm{~min}$, the temperature was raised to $200^{\circ} \mathrm{C}$, and growth of the nano crystals was allowed to proceed at this temperature for $20 \mathrm{~h}$. Under these conditions, tellurium slowly dissolves in the reaction mixture, resulting in a very slow growth of CdTe nano crystals at sufficiently high temperatures. Finally, toluene was added to the warm solution, and the mixture was left for 24 $\mathrm{h}$ at room temperature under stirring. The supernatant liquid CdTe nano crystals dispersed in toluene was separated, collected and then dried for a day. Four samples which are labeled $(\mathrm{a}-\mathrm{d})$ of different sizes were obtained.

The PA measurements were carried out by a gas microphone detection technique. A xenon light beam (400 watt) was focused into the entrance slit of a monochrometer. Then the output beam of the exit slit was mechanically modulated by an optical chopper, and focused onto the sample placed in a closed PA cell. The detected PA signal by high sensitive microphone is lock-in amplified. For thermal properties measurements, the same set-up was used where the xenon light source was replaced by $200 \mathrm{~mW}(514 \mathrm{~nm})$ Argon ion. The PA signal amplitude was measured for each $\mathrm{Cd}$ Te sample. In addition, the 
measurement was repeated several times by keeping the beam intensity and the sample at the same position.

\section{Results and Discussion:}

\subsection{Optical absorption measurements}

The average particle size of these samples were estimated using transmission electron microscope (TEM), which are approximately ranging from $2.10 \mathrm{~nm}$ for sample a to $2.45 \mathrm{~nm}$ for sample d. The TEM micrograph of sample (d) is shown in Fig (1) as an example.

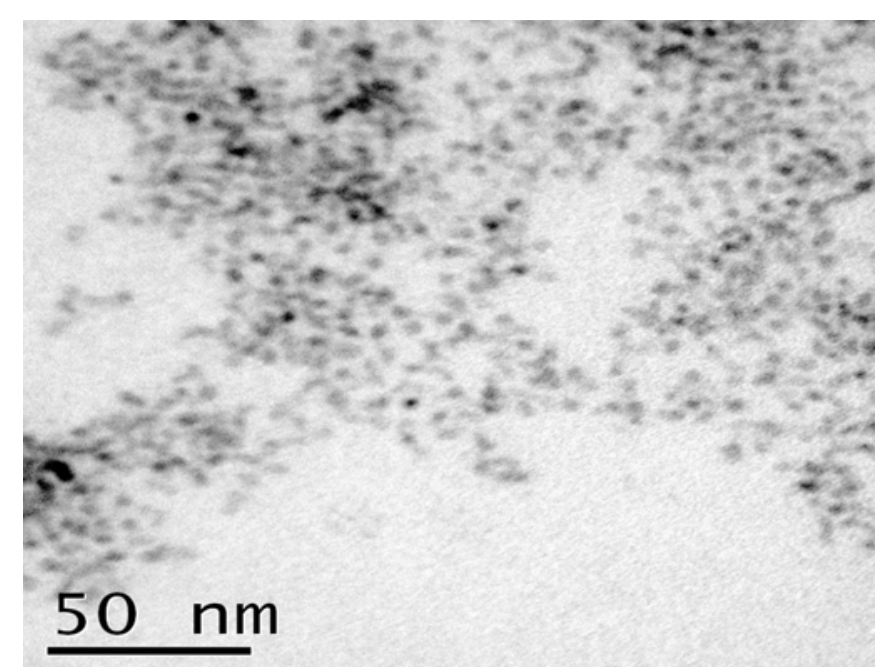

Fig. (1): TEM micrograph of CdTe quantum dots of sample (d).

Fig. 2(a) shows the PA spectra for the four investigated samples of CdTe QDs (a-d) as a function of the wavelength of the incident beam (from $500 \mathrm{~nm}-$ $700 \mathrm{~nm}$ ) for a constant modulation frequency of $15 \mathrm{~Hz}$. The PA spectrum for each sample is normalized using the PA spectrum obtained for carbon black in the allowed region of the used xenon lamp. The absorption edges varies between $540 \mathrm{~nm}$ for sample a to $602 \mathrm{~nm}$ for sample (d). It easily observed that there is a shift towards lower energy region with increasing the size. This behavior is attributed to the quantum confinement effect. The calculated energy band gap $\left(E_{g}=h c / \lambda\right)$ for the same samples varies between $2.3 \mathrm{ev}$ for sample a to $2.09 \mathrm{ev}$ for sample 4 . The optical absorption spectra of the same samples in colloidal solution were also obtained by regular UV-Vis absorption and given in Fig. (2-b). Although, the UV-Vis spectra are for samples in colloidal form, and the PA spectra are for powder form, the two spectra gave peaks that are very close. 


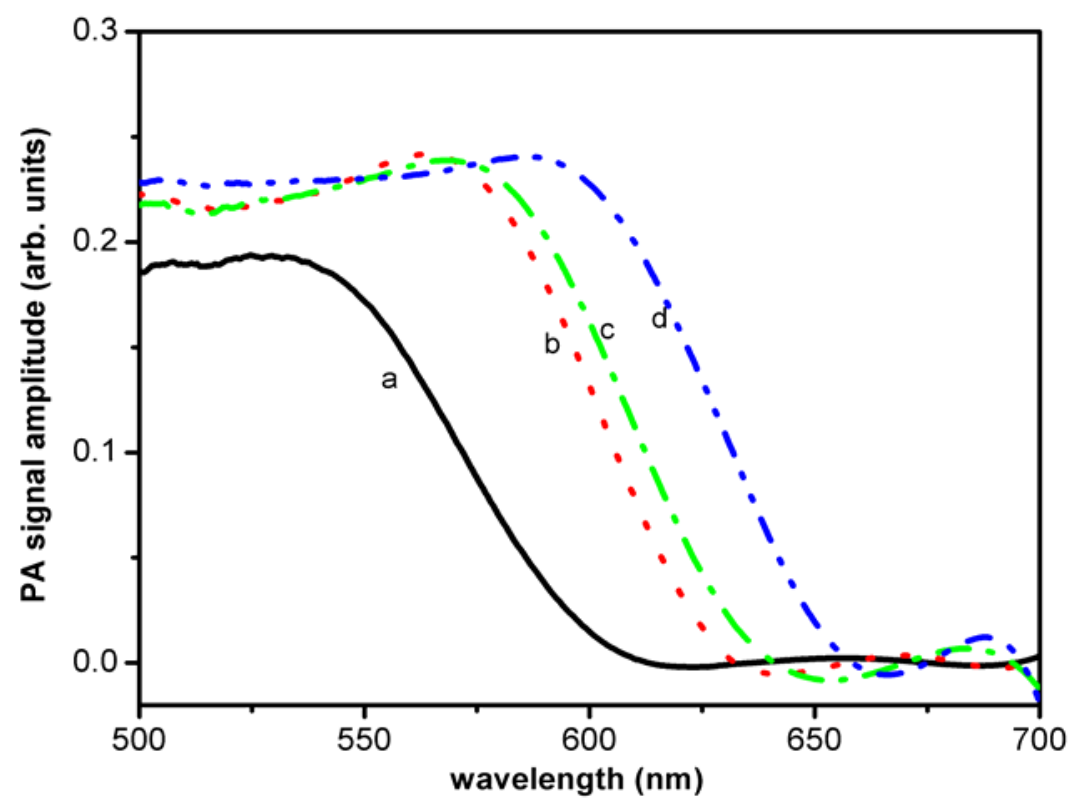

Fig.(2-a) : normalized PA spectra for the four CdTe QDs samples

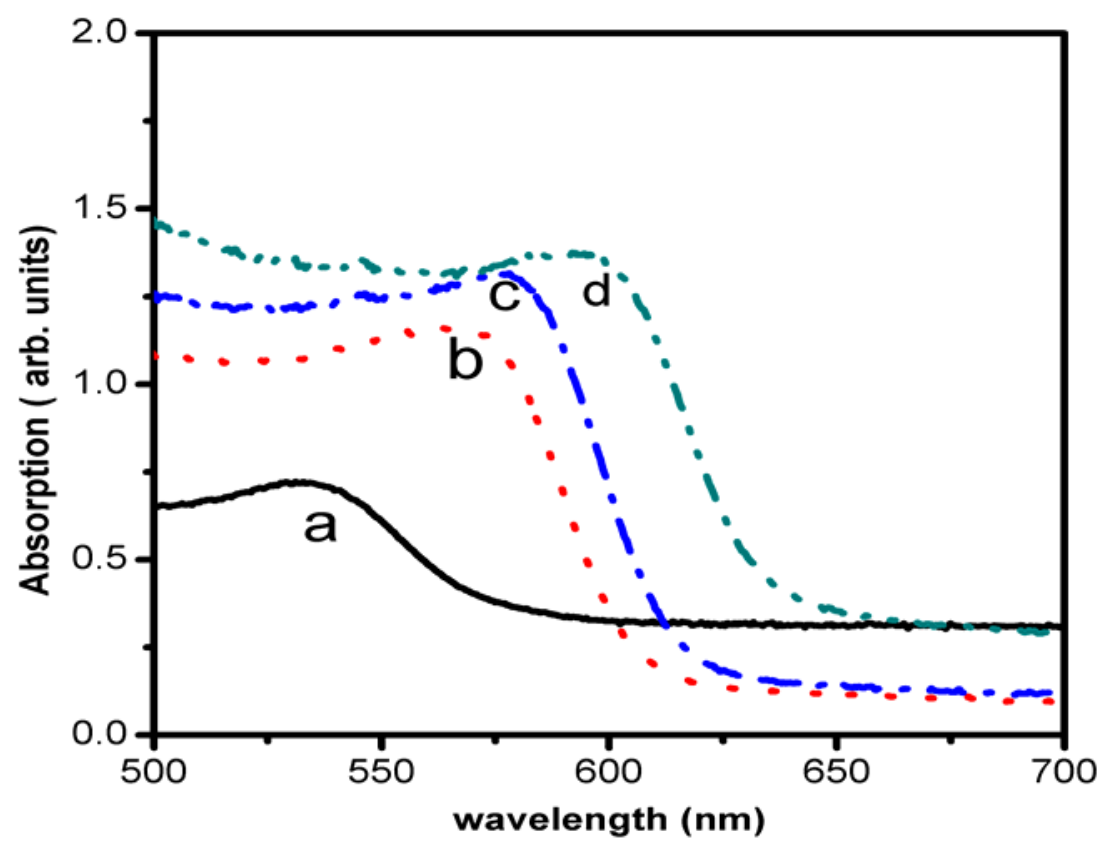

Fig (2-b): UV -Visible absorption spectra for CdTe QDs samples

The size (R) of nano crystal sample can be calculated using the effective mass approximation (EMA) [7, 9] .

$$
E_{g n}(R)=E_{g b}+\frac{h^{2}}{8 m^{*} R^{2}}-\frac{1.8 e^{2}}{4 \pi \varepsilon \varepsilon_{0} R}
$$


where $\mathrm{E}_{\mathrm{gb}}(=1.475 \mathrm{ev})$ is the bulk crystal band gap value [8], $\mathrm{E}_{\mathrm{gn}}$ is the nano crystal band gap value, $\mathrm{R}$ is the radius of the CdTe quantum dots, $\mathrm{m}^{*}(=7.62 \mathrm{x}$ $\left.10^{-32} \mathrm{~kg}\right)$ is the reduced electron-hole mass [13], and $\varepsilon(=7.1)$ is the relative dielectric constant for CdTe [8]. The values of $E_{g n}$ that obtained from PA spectra together with equation 1 are used to calculate the average radii of CdTe QDs. The values of radii are ranging from $2.13 \mathrm{~nm}$ for sample a to $2.43 \mathrm{~nm}$ for sample $d$, which are comparable to those obtained by TEM images. This indicates that the possibility of the regime of individual electron and hole confinement. The values of $\mathrm{E}_{\mathrm{gn}}$ and $\mathrm{R}$ of these samples were also determined using UV-Vis. absorption spectra. The calculated $\mathrm{E}_{\mathrm{gn}}$ from both PA and UVVis. spectra versus R of CdTe QDs are shown in Fig. (3). It is easily observed that the results obtained by both techniques for each sample are very close with a variation $2 \%$.

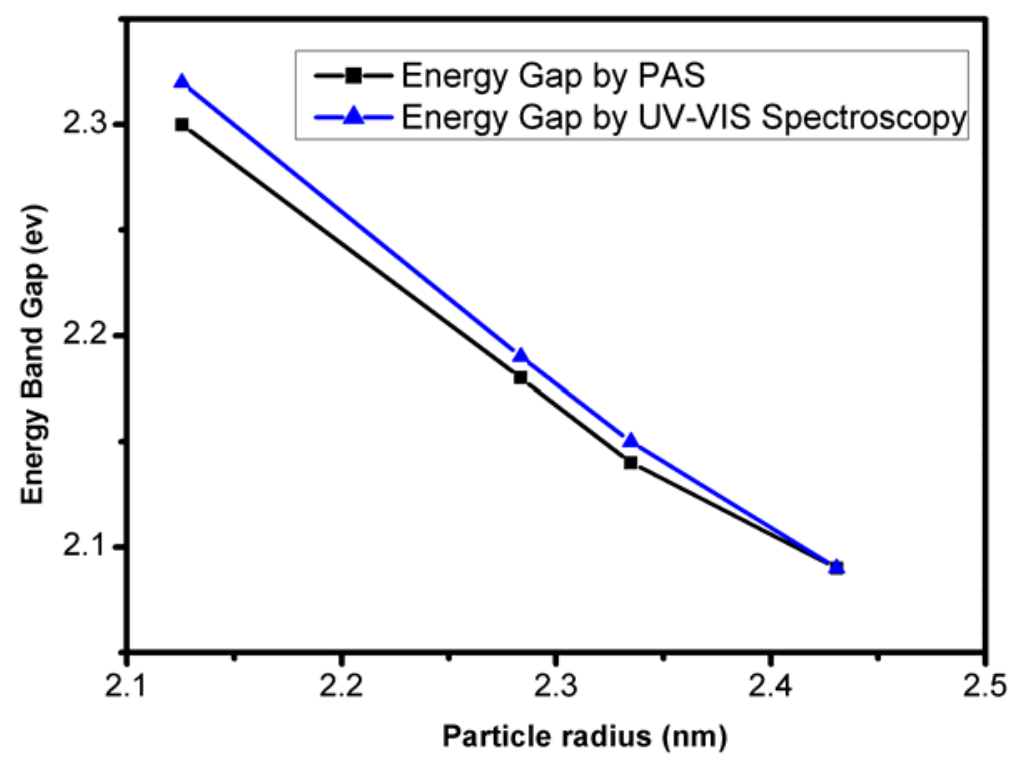

Fig.(3): The values of $\mathrm{E}_{\mathrm{gn}}$ of the $\mathrm{CdTe}$ QDs versus particle radius

\subsection{Thermal properties measurments:}

The PA technique was also employed to investigate the thermal properties of the CdTe QDs. The powder of each sample was compressed (under hydraulic pressure of $1 \mathrm{Ton} / \mathrm{cm}^{2}$ ) into a disk. The PA signal amplitude was recorded at various chopping frequencies (f) for each sample (depth profile analysis). The plots of $\ln$ PA amplitude versus $\ln \mathrm{f}$ are shown in Fig. (4-a) and Fig (4-b) for sample (a) and (d) respectively. A distinct change in the slope is 
clearly observed at a frequency $f_{\mathrm{c}}$ (the sample changes from being thermally thick to thermally thin) where crossover takes place.

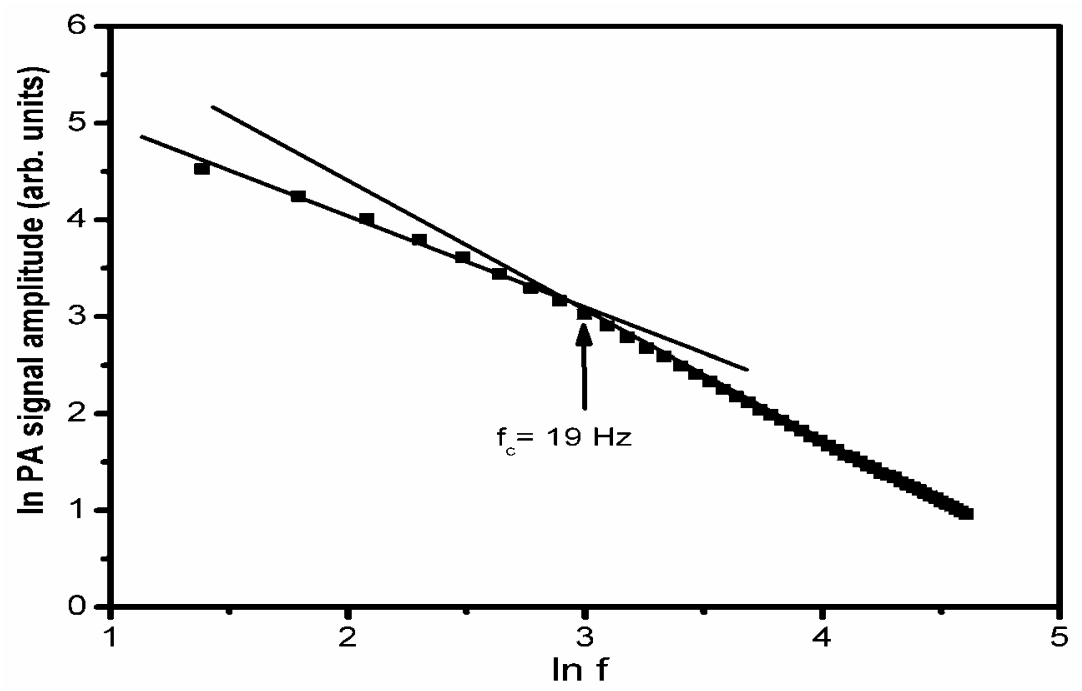

Fig 4(a): Dependence of $\ln$ PA signal amplitude with $\ln \mathrm{f}$ for sample a

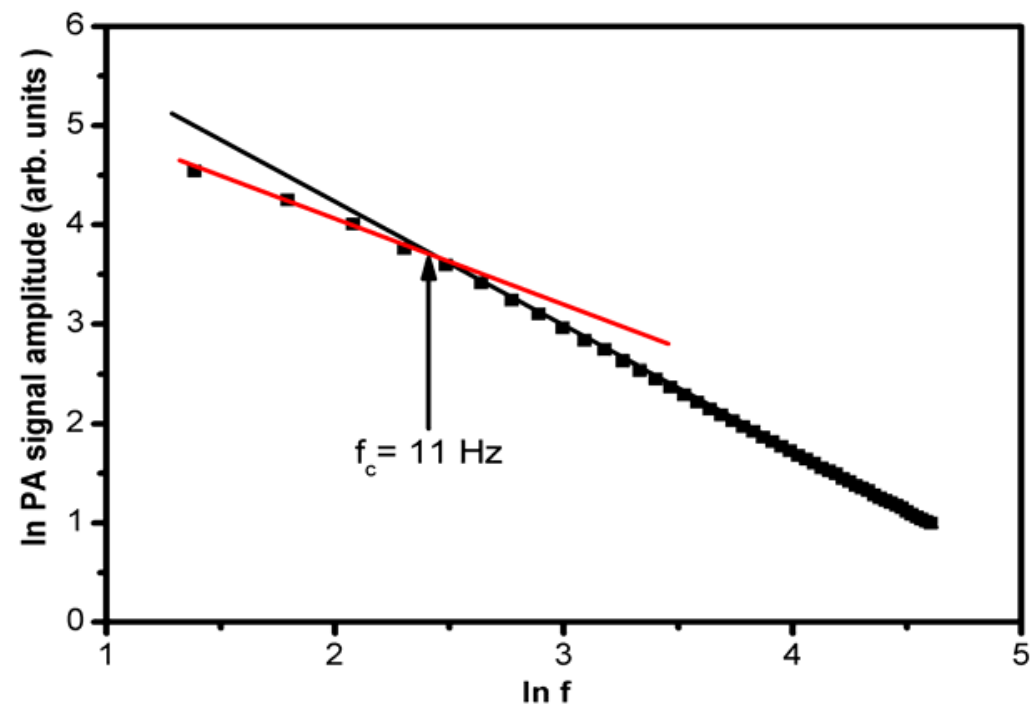

Fig 4(b): dependence of $\ln$ PA signal amplitude with $\ln \mathrm{f}$ for sample $\mathrm{d}$.

The thermal diffusivity (D), for all samples, were then calculated using the relation $[2,10]$. 


$$
D=f_{c} L^{2} \quad \text { in } \mathrm{m}^{2} / \mathrm{s}
$$

where $\mathrm{L}$ is the thickness of the sample. The calculated values of $\mathrm{D}$ are displayed in table (1).The thermal effusivity (e) of the samples were also determined by PA technique, where for optically opaque and thermally thick sample, the PA signal amplitude $\mathrm{q}$ is given by [14].

where

$$
\begin{aligned}
q & =\frac{A}{e} \frac{1}{f} \\
A & =\frac{I_{0} \gamma P_{0} \alpha_{g}^{1 / 2}}{4 \pi l_{g} T_{0}}
\end{aligned}
$$

$\mathrm{I}_{0}$ is the intensity of the incident radiation; $T_{0}$ and $\mathrm{P}_{0}$ are the ambient pressure and temperature respectively, $\gamma$ is the ratio of gas (air) specific heats, $\alpha_{\mathrm{g}}$ is the thermal diffusivity of the gas and $1_{g}$ is the length of the gas column of the PA cell. Using the powder $\mathrm{Si}$ as a standard material of known effusivity $(=16061$ $\mathrm{W} \cdot \mathrm{s}^{1 / 2} \cdot \mathrm{m}^{-2} \cdot \mathrm{K}^{-1}$ ) [8], the constant A was determined and applied in equation (3) to calculate e for CdTe QDs samples. The values of e are given in table (1). The corresponding values of thermal conductivity $\mathrm{k}(=e \sqrt{D})$ are also displayed in Table (1).

Table (1): The calculated values of thermal diffusivity (D), thermal effusivity (e) and thermal conductivity (k) for CdTe QDs samples of different particle sizes.

\begin{tabular}{|c|c|c|c|c|}
\hline $\begin{array}{c}\text { Sample } \\
\text { No. }\end{array}$ & $\begin{array}{c}\text { Radius R } \\
(\mathrm{nm})\end{array}$ & $\begin{array}{c}\text { Thermal diffusivity } \\
(\mathrm{D}) \mathrm{X} 10^{-5} \\
\left(\mathrm{~m}^{2} / \mathrm{s}\right)\end{array}$ & $\begin{array}{c}\text { Thermal } \\
\text { effusivity (e) } \\
\left(\mathrm{Ws}^{1 / 2} \mathrm{~m}^{-2} \mathrm{~K}^{-1}\right) \pm 7\end{array}$ & $\begin{array}{c}\text { Thermal } \\
\text { conductivity ( }) \\
(\mathrm{W} / \mathrm{m} . \mathrm{K})\end{array}$ \\
\hline $\mathrm{a}$ & 2.12 & $6.50 \pm 0.04$ & 12098.53 & $97.54 \pm 0.5$ \\
\hline $\mathrm{b}$ & 2.28 & $5.82 \pm 0.04$ & 11256.30 & $85.87 \pm 0.5$ \\
\hline $\mathrm{c}$ & 2.33 & $5.48 \pm 0.04$ & 8702.15 & $64.42 \pm 0.5$ \\
\hline $\mathrm{d}$ & 2.43 & $3.76 \pm 0.04$ & 7352.04 & $45.08 \pm 0.5$ \\
\hline bulk & ---- & $0.55[11,12]$ & 2643.68 & $6.2[8]$ \\
\hline
\end{tabular}

It is clearly seen, as the size of the QD increases the values of $\mathrm{D}$, e and $\mathrm{k}$ decreases approaches the bulk value. These values are about one order of 
magnitude larger than that of the bulk values. The increase in $\mathrm{D}$ of the nonocrystal compared to the bulk is attributed to; at small particle size, quantum confinement is achieved and the interfaces may form an extremely dense network of paths for fast diffusion through the nanocrystalline material, which made the transport properties in nano systems more rapid than that of the bulk. This increase in D is in reasonable agreement with the results of El-Brolossy et al [2], they found that D of CdSe QDs is one order larger than that of bulk CdSe. Similarly Raji et al. [10] have made measurements on D and $\mathrm{k}$ in the case CdS and reported that two orders of magnitude larger values for nano systems than the bulk value, and a decrease in $\mathrm{D}$ and $\mathrm{k}$ as the particle size increases. They attributed the decrease in the thermal properties $(\mathrm{D}, \mathrm{k})$, as the size of the particle increases, to the reduction in the population in phonons. Otherwise more phonons are not generated and hence less scattering taking place.

\section{Conclusion:}

Using the nondestructive PA technique, we were able to study the optical properties of CdTe QDs of different particle sizes. The spectra shifted to lower energy region with increasing the particle size. Furthermore, the PA technique is also employed to determine the thermophysical parameters $(\mathrm{D}, \mathrm{e}, \mathrm{k})$ of the CdTe QDs of different sizes. The decrease in thermal diffusivity and thermal conductivity with increasing the particle size is attributed to the decrease in the population of phonons.

\section{Acknowledgements:}

The authors wish to thank Taif University for the grant research no. $(1 / 431 / 609)$. The Quantum Optics group at Taif University is also thanked for their assistance during this work.

\section{References}

1. D. Patidar, K. S. Rathore, N. S. Saxena, Kananbala Sharma, T. P. Sharma, Chalcogenide Letters Vol. 5, No. 2, p. 21 (2008).

2. T.A. El-Brolossy, S. Abdallah, T. Abdallah, M.B. Mohamed, S. Negm, and H. Talaat, Eur. Phys. J. Special Topics, 153, 365 (2008).

3. D. K. Dwivedi, Dayashankar \& Maheshwar Dubey, Journal of Ovonic Research, Vol. 5, 35 (2009).

4. Guo-Yu Lan, Zusing Yang, Yang-Wei Lin, Zong-Hong Lin, Hao-Ying Liao and Huan-Tsung Chang, J. Mater. Chem., 19, 2349 (2009). 
5. Dmitri V. Talapin, Stephan Haubold, Andrey L. Rogach, Andreas Kornowski, Markus Haase, and Horst Weller, J. Phys. Chem, B, 105, 2260 (2001).

6. M. Thambidural, N. Murugan, N. Muthukumarasamy, S. Vasantha, R. Balasundaraprabhu, S. Agilan, Chalcogenide Letters Vol. 6, No. 4, p. 171 (2009).

7. Semiconductors : Data Handbook, Springer, Otfried Madelung, ISBN / ASIN: 3540404880.

8. Biljana Pejova, Ivan Grozdanov, Materials Chemistry and Physics 90, 35 (2005).

9. P. Raji, C. Sanjeeviraja, and K. Ramachandran, Cryst. Res. Technol. 39, No. 7, 617 (2004).

10. P. Raji, C. Sanjeeviraja, and K. Ramachandran, Bull. Mater. Sci., Vol. 28, No. 3, 233 (2005).

11. M.A. Gonza'lez-T, A. Cruz-Orea, M. de L. Albor-A F. de L. Castillo- A, Thin Solid Films, 480, 358 (2005).

12. Tito Trindade, Paul O'Brien, and Nigel L. Pickett, Chem. Mater., 13, 3843 (2001).

13. S. Abdallah, Egypt. J. Solids, Vol. 27, No. 2, 251 (2004). 